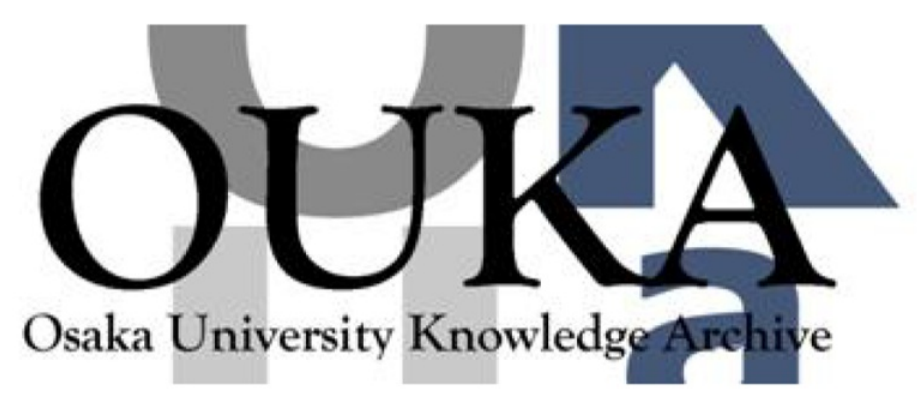

\begin{tabular}{|c|l|}
\hline Title & $\begin{array}{l}\text { Application of Noise-Enhanced Detection of } \\
\text { Subthreshold Signals for Communication Systems }\end{array}$ \\
\hline Author(s) & $\begin{array}{l}\text { Ham, Hyunju; Matsuoka, Toshimasa; Taniguchi, } \\
\text { Kenj i }\end{array}$ \\
\hline Citation & $\begin{array}{l}\text { IEICE Transact ions on Fundamentals of } \\
\text { Electronics, Commun icat i ons and Computer } \\
\text { Sciences. E92-A(4) p. 1012-p. 1018 }\end{array}$ \\
\hline Issue Date & $2009-04$ \\
\hline oaire:version & VoR \\
\hline URL & https://hdl. handle. net/11094/51715 \\
\hline rights & copyright $\odot 2009$ IEICE \\
\hline Note & \\
\hline
\end{tabular}

Osaka University Knowledge Archive : OUKA

https://ir. Library. osaka-u. ac. jp/

Osaka University 


\title{
Application of Noise-Enhanced Detection of Subthreshold Signals for Communication Systems
}

\author{
Hyunju HAM $^{\dagger}$, Nonmember, Toshimasa MATSUOKA ${ }^{\dagger a)}$, and Kenji TANIGUCHI ${ }^{\dagger}$, Members
}

\begin{abstract}
SUMMARY A signal detection system using noise statistical processing is proposed. By approaching the problems of low voltage and high noise from miniaturization of a device from a stochastic point of view, a faint-signal receiving system that can effectively detect subthreshold and noise level signals has been developed. In addition, an alternative to statistical processing is proposed, and would be successfully implemented on a circuit. For the proposed signal detection method, the detection sensitivity was investigated using numerical simulation, and the detection sensitivity was sufficiently high to detect even a signal with a signal-to-inherent-noise ratio of $-14 \mathrm{~dB}$. Thus, it is anticipated that the application of this system to an integrated circuit will have a significant impact on signal processing.

key words: noise, stochastic resonance, statistical processing, communications system
\end{abstract}

\section{Introduction}

Wireless and wired integrated transceivers based on RFCMOS technology have advantages of scalability in size and performance $\left(f_{T}\right.$ and NF). The International Technology Roadmap for Semiconductors (ITRS) [1] predicts that the supply voltages of low-power digital circuits will decrease to $0.5 \mathrm{~V}$ in the near future. Considering the integration of RF and analog circuits with digital circuits, development of receiver circuits operating at such a low supply voltage will be required. However, receiver architectures based on conventional A/D converters are approaching their limit in maintaining resolution under low voltage operation [2], [3]. Even if the A/D converters follow gain stages of amplifiers in receiver chain to maintain resolution, the amplifiers themselves will have difficulty in retaining low nonlinear distortion with enough gain under such a low supply voltage. Therefore, new signal detection techniques to break through this limitation have become an urgent research target.

Although noise-aided detection of weak signals in neurons has been the subject of considerable attention in connection with the phenomenon of stochastic resonance (SR) [4], [5], recent research has also demonstrated SR in nanoscale electron devices, such as carbon nanotube transistors and single-electron devices [6], [7]. As described in a comprehensive review provided by Gammaitoni et al. [5], the SR phenomenon can be generally observed in bistable

\footnotetext{
Manuscript received June 12, 2008.

Manuscript revised November 1, 2008.

${ }^{\dagger}$ The authors are with the Division of Electrical, Electronics and Information Engineering, Osaka University, Suita-shi, 5650871 Japan.

a)E-mail: matsuoka@eei.eng.osaka-u.ac.jp

DOI: 10.1587/transfun.E92.A.1012
}

threshold-based systems that are forced by both of two components: (1) a signal with an amplitude lower than the system threshold, and (2) noise that is inherent in the system. This phenomenon can be interpreted as a reduction in system threshold, which is simply modeled using Brownian particles in a quartic-double-well potential [5]. In terms of applications, improvement of infrared distance sensors using SR in a Schmitt trigger has been demonstrated [8]. Noise-aided techniques to extend the dynamic range of 1bit A/D converters have also been proposed [9].

This paper proposes a novel subthreshold signal detection technique for a communication system, which is suitable for implementation in future nano-scale CMOS technology. Our work firstly applies SR to receiver integrated circuits and reveals its effectiveness as a faint-signal detection technique.

\section{Scheme of Proposed Signal Detection System}

Using an analog comparator as a detection device, it was assumed that the input signal of the comparator is smaller than the noise level, as well as the threshold value. The probability density function of a signal buried in noise $x(t)=$ $s(t)+n(t)$ is given by

$$
p(x)=\frac{1}{\sqrt{2 \pi} \sigma_{n}} \exp \left(-\frac{(x-s)^{2}}{2 \sigma_{n}^{2}}\right),
$$

where $s(t)$ is the input signal, $n(t)$ is the Gaussian white noise, and $\sigma_{n}$ is the standard deviation of the noise. Note that all fluctuating currents and voltages, i.e. noises, generated in electrical devices have Gaussian probability density functions [10]. Thermal and shot noises in electrical devices are typical Gaussian white noises. The probability of detecting a signal with a threshold value $B$ is given by the following equation,

$$
P(x>B)=\frac{1}{2}-\frac{1}{2} \operatorname{erf}\left(\frac{B-s}{\sqrt{2} \sigma_{n}}\right),
$$

where $\operatorname{erf}(y)=(2 / \sqrt{\pi}) \int_{0}^{y} \exp \left(-t^{2}\right) d t$ is an error function. Even if the input signal $s(t)$, lower than the threshold value, cannot be detected itself, the addition of noise enables it to be detected. The input signal $s(t)$ can be restored from the probability $P(x>B)$ using the time or ensemble statistics of the comparator outputs.

In contrast to a distance sensor, which deals with periodic signals [8], [9], random signals are used in communication systems. A signal detection system is proposed 


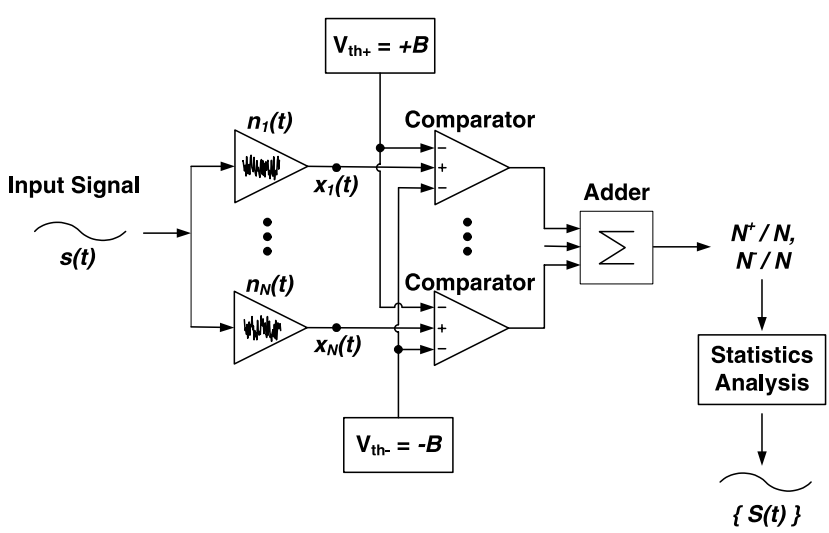

Fig. 1 Schematic of the proposed signal detection system.

that consists of multiple comparators in an array, in order to use the ensemble statistics [4], [7]. This structure allows high-speed signal processing by taking advantage of the integrated circuit. Moreover, the reliability of the signal detection system has been improved using two threshold levels. Figure 1 shows a schematic representation of the proposed system. As an input signal is shared with $N$ amplifiers of the same gain, each output signal $x(t)$ is the sum of the amplified input signal and an inherent noise generated in each amplifier. The comparator then converts the output signal $x(t)$ to a digital signal through comparison with the threshold levels, $+B$ and $-B$. From the $N$ comparator outputs, the number of output signals that are larger than $+B$ is represented as $N^{+}$, and that smaller than $-B$ as $N^{-}$. As a result, two output values, $N^{+}$and $N^{-}$, are obtained from one input signal $s(t)$. The original input signal can be restored by statistical analysis of these two values. In the proposed system, $N^{+} / N$ indicates the probability of signal detection, $P(x>B)$, so that the following relation can be approved.

$$
P(x>B)=\frac{1}{2}-\frac{1}{2} \operatorname{erf}\left(\frac{B-s}{\sqrt{2} \sigma_{n}}\right) \approx \frac{N^{+}}{N} .
$$

The $N^{+} / N$ value does not exactly match the $P(x>B)$. However, the difference between them will be decreased as $N$ increases. Criteria must be set for the acceptable error in the difference between $N^{+} / N$ and $P(x>B)$, and this will be discussed in more detail in Sect. 4 . Therefore, the signal can be restored using the inverse calculation of Eq. (3) with the statistical analysis blocks as follows.

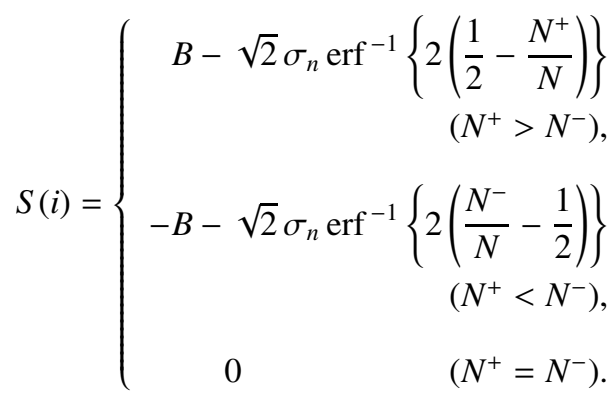

Here $S(i)$ represents the sample value of $s(t)$ at $t=i T_{s}(i=$ $0,1,2, \cdots)$, where $T_{s}$ is the sampling period.

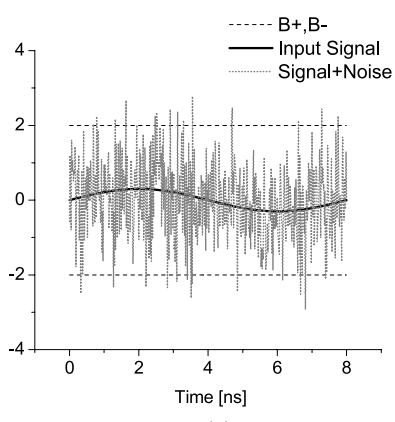

(a)

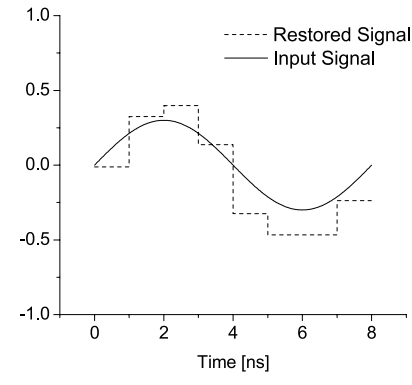

(b)
Fig. 2 (a) Test conditions in each comparator, where $A_{s}=0.3$ is the amplitude of the input signal, $\sigma_{n}=1, B=2$, and $N=128$. (b) Restoration using the proposed system. Over sampling rate $(\mathrm{OSR})=8$. (The vertical axis has no unit because it represents the relative value)

Figure 2 shows an example of signal restoration using the proposed method. Using an array of 128 comparators, a signal can be restored with $95.1 \%$ correlation to the initial input signal. It was also confirmed that the proposed method can substitute Schmitt trigger circuits for comparators. In addition, improved performance in signal restoration is expected when combined with $q$-bit A/D converter $(q>2)$. In other words, the proposed technique can give super-resolution feature beyond the LSB of the conventional $q$-bit A/D converters. This feature is useful for high-speed A/D converters with low-to-moderate resolution which can have scaling merits easily.

Although the Gaussian white noises are used in the proposed system as described above, we use only Gaussian probability density functions of the noises and their independent behaviors. Frequency spectrum of the noises does not influence fundamental behavior of the proposed system. The numerical simulation (not shown) reveals the $1 / f$ Gaussian noises also can have the same role in the proposed system. However, over-sampling effect on $1 / f$ noises in the proposed system differs from that on white noises, which originates from low pass filtering in over-sampling. To simplify analytical approaches for design methodology of the proposed system, only Gaussian white noises are considered in this work. This is not so fatal assumption because $1 / f$ noise can be reduced significantly using some circuit techniques such as correlated double sampling and chopper stabilization [11].

\section{Criteria on Detection Sensitivity}

In order to estimate the detection sensitivity or limit of the proposed system, the establishment of assumptions for the numerical simulation and methods to evaluate the reliability of the restored signal are required.

\subsection{Assumptions}

The following three assumptions were used in the simulation.

- The noise follows a Gaussian probability density func- 
tion and is independent of that from the other amplifiers.

- Each inherent noise is represented as the inputreferred-noise of each amplifier.

- The gain of each amplifier is set to unity.

The phase-shift-keying (PSK) modulated signal, which is widely used in wireless communications, was used as the input signal in this study. The inherent noise can be determined by the circuit design, because the noise used in this system is directly generated by the device itself without the addition of other intentional noise [8] or noise dithering. Thus, the detection sensitivity and the optimal thresholdto-noise ratio were investigated. Setting the amplifier gain at unity is useful to decrease the number of parameters required. Once the sensitivity of the proposed system is characterized, the value of gain will be readjusted, taking into consideration the required sensitivity and the inherent noise.

\subsection{Method to Evaluate Reliability of Signal Restoration}

To evaluate the reliability of the system output for signal restoration, the following criteria were established.

- The digital data represented by the restored signal are determined by the correlation of the output and the signal pattern modulated by the corresponding digital data.

- Bit error rate (BER) less than or equal to $10^{-3}$ is required for reliable signal detection in communications.

The PSK modulated signal used as an input signal is represented by

$$
\begin{aligned}
& s(t)=A_{s} \cos \left(2 \pi f_{c} t+\alpha_{m} \Delta \Theta\right) \\
& \alpha_{m}=\{0,1, \cdots, m-1\} \\
& \Delta \Theta=\frac{2 \pi}{m},
\end{aligned}
$$

where $s(t)$ is $m$-PSK modulated signal and $f_{c}$ is the carrier frequency. The values of $\alpha_{m}$ are changed according to the random data of the transmitter. In this simulation, the digital data received can be determined by searching the modulated signal pattern with the best correlation to the restored signal. The received data for the restored signal are reliable when the BER is less than or equal to $10^{-3}$ for 10,000 test repetitions under the same conditions.

\section{Sensitivity of Proposed Receiver System}

The detection sensitivity for the subthreshold signal is represented as $A_{s, \min } / B$, which is normalized by the threshold $B$. Smaller $A_{s, \min } / B$ means better detection sensitivity. The sensitivity was investigated for various threshold values under a constant noise level. In addition, the effects of the number of arrayed comparators, the over-sampling ratio (OSR), and the modulation type of input signal on the sensitivity were investigated. Noteworthy points in the simulation were the optimal ratio of $B / \sigma_{n}$ and the subsequent sensitivity.
Firstly, the effect of the number of arrayed comparators, $N$, was considered. Better performance is expected with an increased number of $N$, because the distribution of $N^{+} / N$ becomes narrow with increased $N$, and closer to the theoretical probability, $P(x>B)$, as explained by the law of large numbers. The samples of each comparator were independent random variables with identical distribution and finite expected values. The following relation can be induced.

$$
\delta_{Y}=\sqrt{\overline{(Y-\bar{Y})^{2}}}=\delta / \sqrt{N},
$$

where $Y$ and $\delta^{2}$ are the ensemble average $\left(N^{+} / N\right)$, and the variance of the comparator outputs, respectively. Equation (6) shows that the sensitivity is improved with increasing $N$ in proportion to $1 / \sqrt{N}$. The effect of OSR on the detection sensitivity can be interpreted in a similar way, except for the fact that the spatial increase of samples $(N)$ switched to a temporal increase of samples. Therefore, the detection sensitivity can be expressed as a function of $N$ and OSR, according to the following proportional equation.

$$
\frac{A_{s, \min }}{B} \propto \frac{1}{\sqrt{O S R \times N}}
$$

Next, the modulation type also affects detection sensitivity. For example, the baseband signal power per bit in Quadrature Phase-Shift-Keying (QPSK) is theoretically $3 \mathrm{~dB}$ smaller than that in Binary Phase-Shift-Keying (BPSK). Based on the energy per bit, the detection sensitivity in $m$-PSK can be extended to the following expression.

$$
\frac{A_{s, \min }}{B} \propto \sqrt{\frac{m}{O S R \times N}}
$$

The above result was checked with the numerical simulation, and the influence of parameter mismatches and common noise were also investigated.

\subsection{Detection Sensitivity Simulation}

Firstly, the optimal threshold value was investigated. Figure 3 shows that the resulting optimal ratio of $B / \sigma_{n}$ is moving from 2 to 1 for the increase of $N$. It is desirable that the

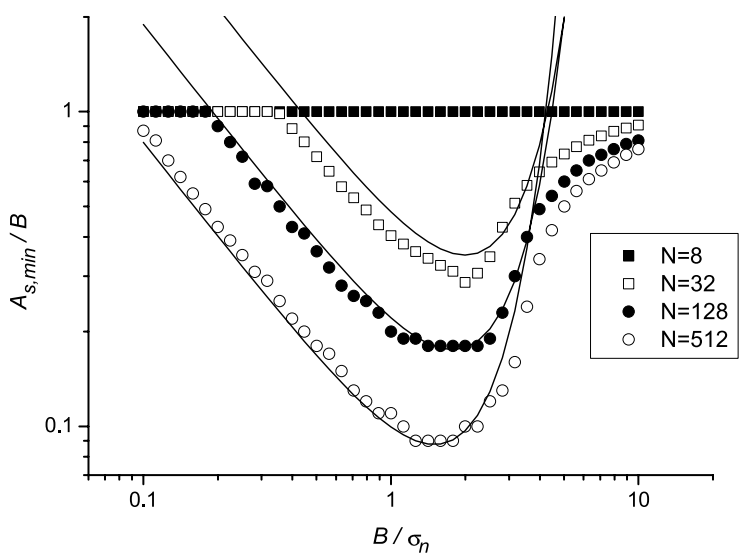

Fig. 3 Detection sensitivity $A_{s, \min } / B$ as a function of $B / \sigma_{n}(O S R=8$, BPSK). The solid lines show the data approximated by Eq. (10). 


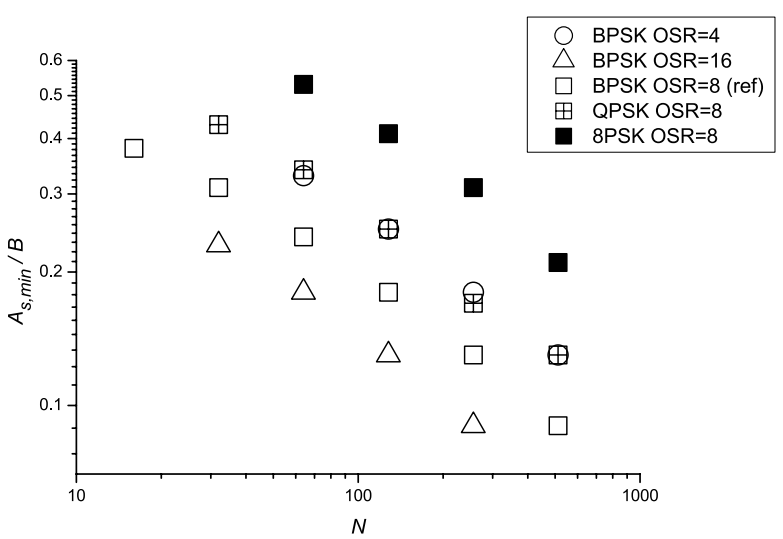

Fig. 4 Detection sensitivity $A_{s, \min } / B$ as a function of $N, O S R$ and $m$-PSK $\left(B / \sigma_{n}=1.77\right)$.

value of $|P(x>B)-P(x<-B)|$ becomes the maximum for optimal ratio of $B / \sigma_{n}$. So that, the following equation can be induced.

$$
\left(B / \sigma_{n}\right)_{\text {ideal }}=1,
$$

where $\left(B / \sigma_{n}\right)_{\text {ideal }}$ is the optimal ratio of $B / \sigma_{n}$ for large $N$ and small $s(t)$. The moving of optimal ratio of $B / \sigma_{n}$ is explained by Eq. (9). The deduction of Eq. (9) is described in Appendix A. As seen in Fig. 3, the increase of $N$ can enhance the detection sensitivity and also provide a slightly wider margin for the optimal threshold value. This behavior is similar to those in many SR systems [4]-[7]. In the case of optimizing $B / \sigma_{n}, B$ should be smaller to enhance the absolute detection sensitivity $A_{s, \min }$.

The numerical simulation results shown in Fig. 3 can be approximated by

$$
\frac{A_{s, \min }}{B}=\frac{P}{Q} \sqrt{\frac{m}{O S R \times N}} \frac{\sigma_{n}}{B} \exp \left(Q^{2} \frac{B^{2}}{2 \sigma_{n}^{2}}\right),
$$

where $P=3.4$ and $Q=\left(\sigma_{n} / B\right)_{\text {opt }}$ are fitting parameters. The deduction of Eq. (10) is described in Appendix B. For large $N$, Eq. (10) can explain the detection behavior very well.

Next, the detection sensitivity $A_{s, \min } / B$ was simulated for various $N$, OSR, and modulation types. Figure 4 reveals the enhancement in detection sensitivity with the increase of $N$ and OSR. This indicates that the performance of the proposed architecture can be improved with scaling-down of the integrated circuit. The detection sensitivities for the QPSK and 8PSK modulated input signals differ from that for BPSK by a factor $\sqrt{2}$ and 2 , respectively. This means that OSR or $N$ should be doubled and quadrupled for the QPSK and 8PSK input signals, respectively, to achieve the same sensitivity as that for BPSK. The above results match the theoretical formula of Eq. (8).

\subsection{Influence of Parameter Mismatches}

As device parameter mismatches in the chip limit the resolution of many analog integrated circuits [3], acceptable parameter mismatches were investigated for the proposed

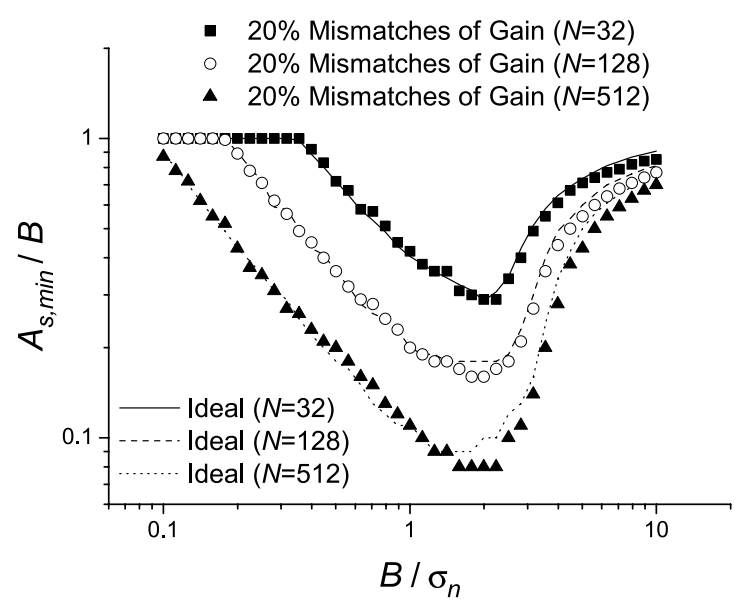

(a)

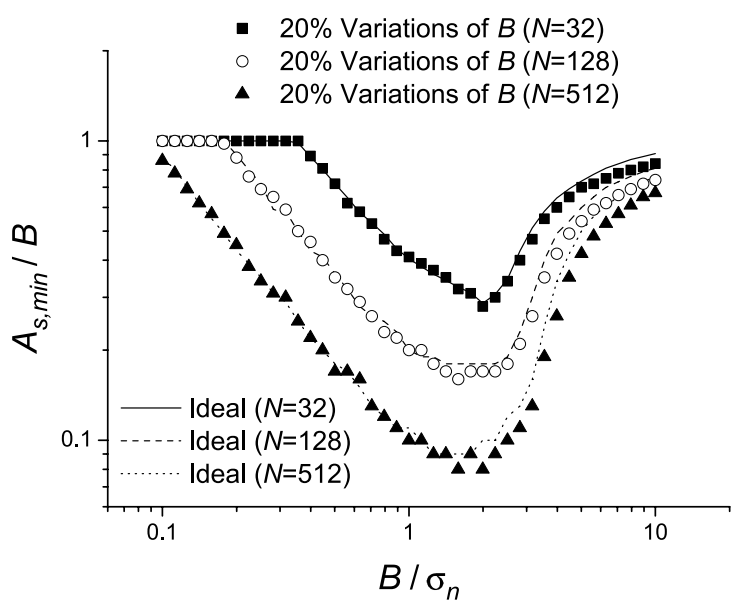

(b)

Fig. 5 Detection sensitivity with consideration of the variation in: (a) gain, and (b) threshold ( $O S R=8$, BPSK).

signal detection system. In the proposed system, the mismatch of each arrayed circuit can be evaluated equally as DC noise. Thus, the total noise and device mismatch can be expressed as

$$
\sigma_{\text {total }}=\sqrt{\sigma_{n}^{2}+\sigma_{\text {th }}^{2}+\sigma_{\text {gain }}^{2} A_{s}^{2} / G^{2}},
$$

where $\sigma_{n}, \sigma_{t h}$ and $\sigma_{\text {gain }}$ are the standard deviations of noise, comparator threshold $B$, and amplifier gain $G$, respectively. The mismatches of threshold and gain approximately follow the Gaussian distribution, which is based on experimental device mismatch data [12], [13].

The numerical simulation results shown in Fig. 5 reveal no performance degradation in the proposed signal detection system, even for $20 \%$ parameter mismatches, and even show sensitivity improvement in the case of high threshold. These results reflect that the parameter mismatches are effectively equivalent to the DC noise.

The mismatches of $\sigma_{n}$ among the arrayed circuits also exist. However, Eq. (10) shows the sensitivity $A_{s, \min } / B$ is influenced by the ratio $\sigma_{n} / B$. The mismatches of $\sigma_{n} / B$ are 


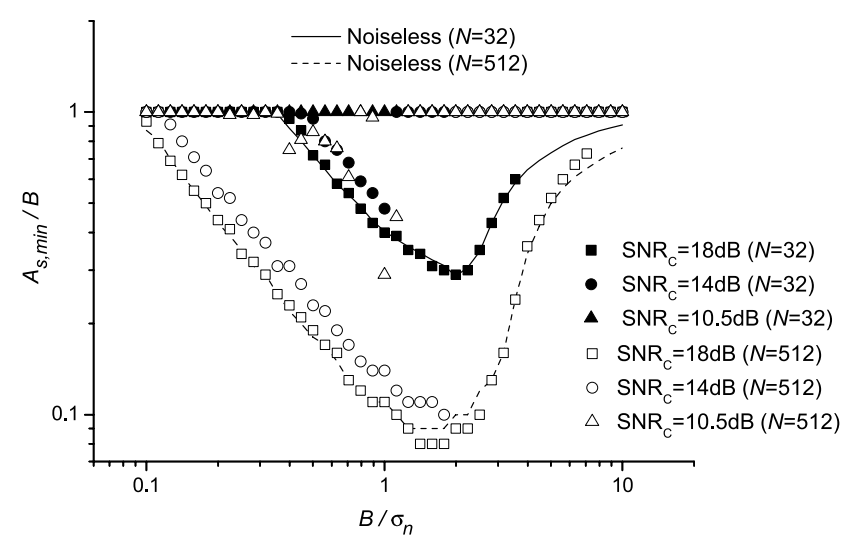

Fig. 6 Behavior of detection sensitivity $(O S R=8$, BPSK) as a parameter of input signal-to-common-noise ratio $\left(\mathrm{SNR}_{\mathrm{C}}\right)$.

expressed as follows.

$$
\frac{\Delta\left(\sigma_{n} / B\right)}{\sigma_{n} / B}=\frac{\Delta \sigma_{n}}{\sigma_{n}}-\frac{\Delta B}{B}
$$

This means the mismatches of $\sigma_{n}$ among arrayed circuits are equivalent to those of $B$ except for their signs.

\subsection{Influence of Common Noise}

The acceptable noise level for the common signal path in front of the arrayed amplifiers was considered. This common noise cannot be separated from the input signal using noise statistics, because it differs from the independent noise among the arrayed amplifiers. As a result, the common noise directly degrades the signal to noise ratio (SNR). Figure 6 shows little sensitivity degradation in the case of $\mathrm{SNR}_{\mathrm{C}}=18 \mathrm{~dB}$. However, in the case of $\mathrm{SNR}_{\mathrm{C}}=10.5 \mathrm{~dB}$, the signal detection sensitivity is largely degraded, even for $N=512$. Therefore, it is highly necessary to reduce the common input noise as much as possible. For example, the use of a filter or low noise amplifier (LNA) is recommended.

\section{Circuit Implementation of Statistical Processing Block}

To implement the proposed system in an integrated circuit, the statistical processing block that follows the digital adder is one of the most serious issues. As statistical processing uses an inverse error function, it is almost impossible to implement it in a real-time processing circuit. The following equations approximate well within the entire range used in the proposed system.

$$
\begin{aligned}
& \operatorname{erf}(y) \approx\left\{1-\exp \left(-\frac{4 y^{2}}{\pi}\right)\right\}^{\frac{1}{2}} \\
& \operatorname{erf}^{-1}(y) \approx \frac{\sqrt{\pi}}{2}\left\{\log \left(\frac{1}{1-y^{2}}\right)\right\}^{\frac{1}{2}}
\end{aligned}
$$

These approximations reduce the numerical simulation running time by $1 / 3$. However, even use of these approxima-

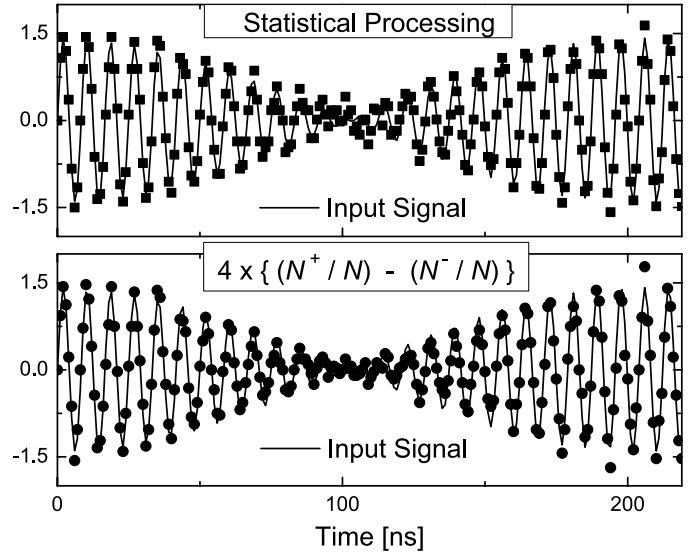

Fig. 7 Waveforms of restored signals by statistical processing and $4\left(N^{+} / N-N^{-} / N\right)$. The input signal has two tones $\left(f_{\text {in } 1}=125 \mathrm{MHz}, f_{\text {in } 2}\right.$ $=117.1875 \mathrm{MHz}) . A_{s}=0.711, \sigma_{n}=1, B=1.7783, N=128, f_{\text {sample }}=$ $1 \mathrm{GHz}$.

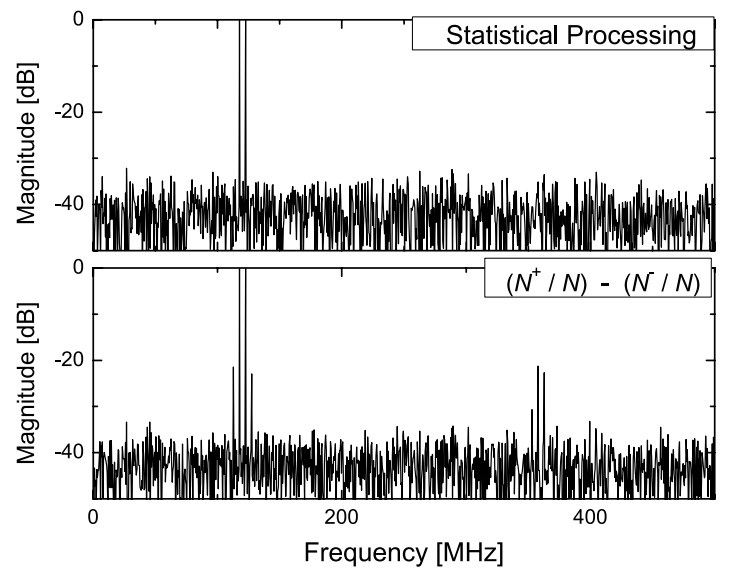

Fig. 8 Frequency spectrum of two-tone restored signals by statistical processing and by the proposed restoration $\left(N^{+} / N-N^{-} / N\right)$.

tions is difficult to implement in a real-time processing circuit.

To implement the real-time statistical processing circuit, use of the difference between the two adder outputs, $\left(N^{+} / N-N^{-} / N\right)$, is proposed for simplicity. The technique based on Eq. (4), uses only one of $N^{+} / N$ and $N^{-} / N$, while the proposed approach considers both $N^{+} / N$ and $N^{-} / N$ simultaneously. Figures 7 and 8 show the comparison of signal restoration for a two-tone input signal. Figure 7 shows that the relative waveform of the input signal is also well restored. Considering the three-level output of the comparators, this result also shows suppression of the quantization error. Figure 8 shows a similar SNR can be available, although the proposed method shows slight nonlinear distortion for two-tone input compared to that for the statistical processing. Furthermore, note that use of Schmitt trigger circuits instead of comparators can eliminate evaluation of the difference of the two outputs $\left(N^{+} / N-N^{-} / N\right)$ in the adding process.

Figure 9 shows intermodulation distortion (IMD) of 


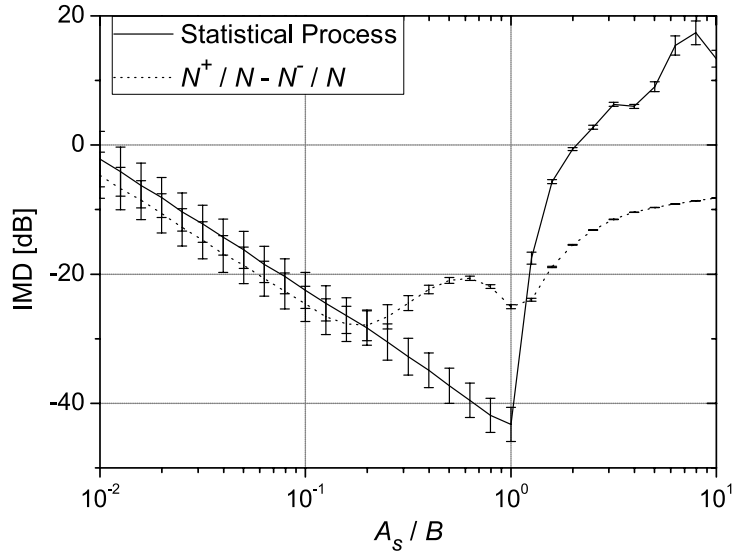

Fig. 9 Intermodulation Distortion (IMD) of two-tone restored signals by statistical processing and by the proposed restoration $\left(N^{+} / N-N^{-} / N\right)$ as a function of $A_{s} / B$. Error bars mean standard deviations for 1000 trials.

two-tone restored signals by statistical processing and by the proposed restoration $\left(N^{+} / N-N^{-} / N\right)$ as a function of $A_{s} / B$. The values of IMD contain the third-, fifth- and seventhorder distortions. In small $A_{s} / B$ region, decrease of IMD means just increase of fundamental frequency component of signal because the power of distortions are below noise floor. In the case of the statistical processing, IMD increases with $A_{s} / B$ for $A_{s} / B>1$. On the other hand, the proposed simplified processing shows IMD increase for smaller $A_{s} / B$. For receiver systems with severe intermodulation distortion requirements, more accurate statistical processing must be developed.

\section{Simulation for Circuit Implementation}

Using the results obtained so far, a numerical simulation was performed to evaluate the applicability of this system as a receiver circuit, as shown in Fig. 10. The bandpass filter at the input stage maintains $\mathrm{SNR}_{\mathrm{C}}$ greater than $20 \mathrm{~dB}$. The LNA in front of the 128 arrayed blocks amplifies the signal suppressing increase of the common noise. The BER was calculated by changing the input amplitude from $1 \mu \mathrm{V}$ to $100 \mu \mathrm{V}$, and the results are shown in Fig. 11. The proposed signal detection system is expected to restore even small input signals of $20 \mu \mathrm{V}$, which is equivalent to subthreshold signal power of 0.04 times $(-14 \mathrm{~dB})$ of inherent noise power, with BER $=10^{-3}$.

\section{Conclusion}

A signal detection system was proposed for small signals below the threshold using SR. The structure of the arrayed comparators realizes high-speed signal processing. Use of two-level comparators can realize the simple implementation of real-time statistical processing without any serious performance degradation. The dynamic range of a receiver circuit for communication can be significantly improved by application of the proposed system. A subthreshold signal power of 0.04 times $(-14 \mathrm{~dB})$ of inherent noise power,

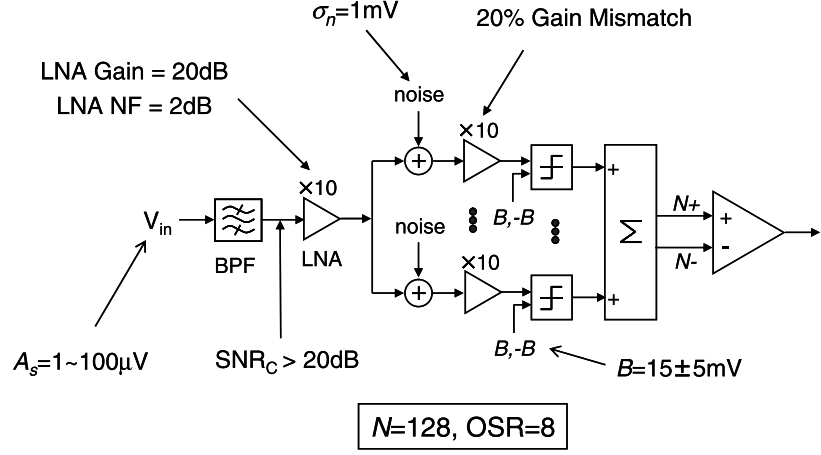

Fig. 10 Application to receiver circuit.

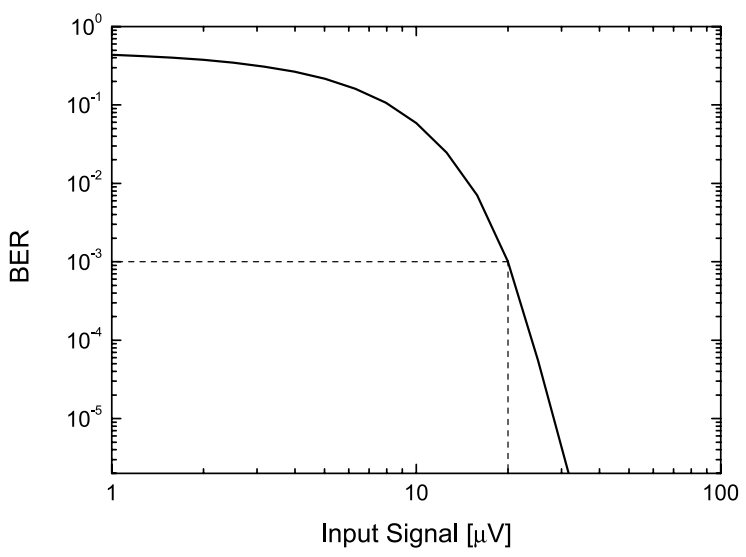

Fig. 11 BER characteristics $(O S R=8$, BPSK).

which is equivalent to a signal amplitude of $20 \mu \mathrm{V}$, is restored with BER $=10^{-3}$.

The proposed technique can have superior detection sensitivity beyond conventional A/D-converter based CMOS receivers, and provide a solution for maintaining resolution under low voltage operation.

As this study is the early generation of its kind, there is much room for study, such as circuit design and comparison with conventional A/D-converter based systems in speed, size, and power dissipation. However, it is our belief that the proposed technique will provide a new design paradigm for wireless and wired receivers.

\section{Acknowledgments}

This work is supported by the Industrial Technology Research Grant Program in 2006 from the New Energy and Industrial Technology Development Organization (NEDO) of Japan. In addition, this work is also partially supported by a grant to the Osaka University Global COE Program, "Center for Electronic Devices Innovation," from the Ministry of Education, Culture, Sports, Science and Technology of Japan.

\section{References}

[1] Semiconductor Industry Association, "International technology 
roadmap for semiconductors 2007 edition," Available:http://www. itrs.net/

[2] R.H. Walden, "Analog-to-digital converter survey and analysis," IEEE J. Sel. Areas Commun., vol.17, no.4, pp.539-550, April 1999.

[3] K. Uyttenhove and M.S.J. Steyaert, "Speed-power-accuracy tradeoff in high-speed CMOS ADCs," IEEE Trans. Circuits Syst. II, vol.49, no.4, pp.280-287, April 2002.

[4] J.J. Collins, C.C. Show, and T.T. Imhoff, "Stochastic resonance without tuning," Nature, vol.376, pp.236-238, July 1995.

[5] L. Gammaitoni, P. Hänggi, P. Jung, and F. Marchesoni, "Stochastic resonance," Rev. Mod. Phys., vol.70, no.1, pp.223-287, Jan. 1998.

[6] I. Lee, X. Liu, C. Zhou, and B. Kosko, "Noise-enhanced detection of subthreshold signals with carbon nanotubes," IEEE Trans. Nanotechnol., vol.5, no.6, pp.613-627, Nov. 2006.

[7] T. Oya, T. Asai, and Y. Amemiya, "Stochastic resonance in an ensemble of single-electron neuromorphic devices and its application to competitive neural networks," Chaos, Soliton and Fractals, vol.32, pp.855-861, 2007.

[8] B. Andò and S. Graziani, "Adding noise to improve measurement," IEEE Instr. \& Meas. Magazine, vol.4, no.1, pp.24-31, March 2001.

[9] G. Tapang and C. Saloma, "Dynamic-range enhancement of an optimized 1-bitA/D converter," IEEE Trans. Circuits Syst. II, vol.49, no.1, pp.42-47, Jan. 2002.

[10] A. van der Ziel, Noise in Solid State Devices and Circuits, John Wiley \& Sons, Toronto, 1986.

[11] R. Gregorian and G.C. Temes, Analog MOS Integrated Circuits for Signal Processing, pp.500-513, John Willy \& Sons, 1986.

[12] Y. Shimizu, M. Nakamura, T. Matsuoka, and K. Taniguchi, "Test structure for precise measurement of MOSFET matching properties," IEICE Trans. Electron. (Japanese Edition), vol.J86-C, no.7, pp.726-733, July 2003.

[13] Y. Shimizu, M. Nakamura, T. Matsuoka, and K. Taniguchi, "Test structure for precise measurement of MOSFET matching properties," Electron. Commun. Jpn. 2, Electron., vol.87, no.3, pp.21-28, March 2004.

\section{Appendix A}

For small $s(t),|P(x>B)-P(x<-B)|$ is approximated by

$$
\begin{aligned}
\mid P(x>B)- & P(x<-B) \mid \\
& \approx 2 s \times \frac{1}{\sqrt{2 \pi} \sigma_{n}} \exp \left(-\frac{B^{2}}{2 \sigma_{n}^{2}}\right)
\end{aligned}
$$

Equation (9) can be deduced by the value of $\sigma_{n}$ at which Eq. (A. 1) becomes maximum for fixed $B$.

\section{Appendix B}

Equation (10) can be deduced from Eq. (3). For small $s(t)$, Eq. (3) is approximated by

$$
\begin{aligned}
P(x>B) \approx & \frac{1}{2}-\frac{1}{2} \operatorname{erf}\left(\frac{B}{\sqrt{2} \sigma_{n}}\right) \\
& +\frac{s(t)}{\sqrt{2 \pi} \sigma_{n}} \exp \left(-\frac{B^{2}}{2 \sigma_{n}^{2}}\right) .
\end{aligned}
$$

The last term depending on signal must be larger than a critical value $p_{c r}$ to achieve BER requirement. Therefore, the detection sensitivity $A_{s, \min }$ can be given by

$$
A_{s, \min } \approx \sqrt{2 \pi} p_{c r} \frac{\sigma_{n}}{Q} \exp \left(Q^{2} \frac{B^{2}}{2 \sigma_{n}^{2}}\right),
$$

where noise effect through the nonlinearity of comparators is considered by standard deviation of effective noise $\sigma_{n} / Q$ with a fitting factor $Q$. Based on Eq. (8) and Eq. (A-3), Eq. (10) can be deduced with another fitting parameter $P$ related to $p_{c r}$.

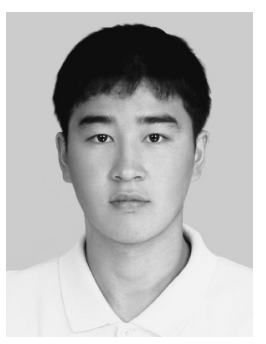

Hyunju Ham was born in Ulsan, Korea, in 1983. He received the B.S. degree in electronic engineering in 2007. He is currently pursuing the M.S. degree at Osaka University, Japan. His current research interests are analog-to-digital conversion.

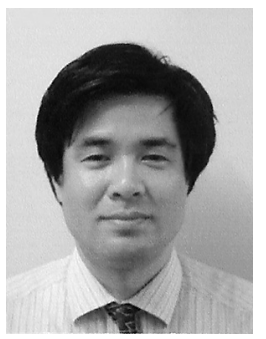

Toshimasa Matsuoka received the B.S., M.S. and Ph.D. degrees in electronic engineering from Osaka University, Osaka, Japan, in 1989, 1991 and 1996, respectively. During 1991-1998, he worked for the Central Research Laboratories, Sharp Corporation, Nara, Japan, where he was engaged in the research and development of deep submicron CMOS devices and ultra thin gate oxides. Since 1999, he has been working for Osaka University, where he is Associate Professor now. His current research includes CMOS RF circuits and device modeling. Dr. Matsuoka is a member of the Japan Society of Applied Physics, the IEEE, and the IEEJ.

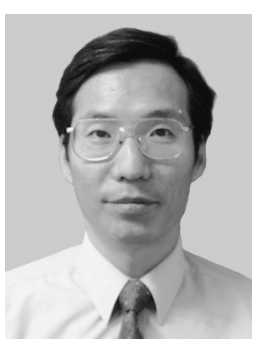

Kenji Taniguchi received the B.S., M.S. and Ph.D. degrees from Osaka University, Osaka, Japan, in 1971, 1973 and 1986 respectively. From 1973 to 1986, he worked for Toshiba Research and Development Center, Kawasaki, Japan, where he was engaged in process modeling and the design of MOS LSI fabrication technology. He was a Visiting Scientist at Massachusetts Institute of Technology, Cambridge, from July 1982 to November 1983. Presently, he is a Professor of Electronics Engineering at Osaka University. His current research interests are in analog circuits, radio frequency circuits, device physics and process technology. Prof. Taniguchi is a member of the Japan Society of Applied Physics and the IEEJ. He is a fellow of the IEEE. 